\title{
ANÁLISIS SOCIAI. DE LAS FAMIIIIAS DEL BARRIO NELSÓN MANDELA DE CARTAGENA DE INDIAS, EN CUANTO A SUS CONOCIMIENTOS EN SALUD Y ENFERMEDAD GENERAL Y ORAL
}

\author{
${ }^{1}$ Farith González M., ${ }^{2}$ Luis Carmona A., ${ }^{3}$ Yaireth Agudelo, ${ }^{3}$ Paula Campo. \\ ${ }^{\prime}$ Coordinador Departamento de Investigaciones $F$ de Odontología, U. de Cartagena. Especialista en Investigación Social, Aspirante a \\ Maestría en Salud Pública ${ }^{2}$ Especialista en Odontopediatría, Docente F de Odontología, U. de Cartagena, Especialista en \\ Odontopediatría. Director grupo "PROMOUC," ${ }^{3}$ Estudiante de X Semestre, F. de Odontología, U. de Cartagena
}

Autor responsable de correspondencia: Farith González Martínez. Dirección de correo electrónico: farithgm@hotmail.com

\begin{abstract}
RESUMEN
Objetivo: Analizar los conocimientos en salud y enfermedad oral que tiene la población de Nelson Mandela en Cartagena de Indias, teniendo en cuenta la situación social en la que se encuentran.

Materiales y métodos: Enfoque metodológico cualitativo, basado en el método hermenéutico, implementado en la población de Nelson Mandela. Se seleccionaron diez familias de estrato 1 y 2 correspondientes al núcleo familiar de niños escolarizados en edades de 2 y 19 años. Para la recolección de la información se utilizó la entrevista individual y la observación no participante en las personas responsables del cuidado de los niños, seleccionando las interacciones de mayor interés para los objetivos del estudio.

Resultados: Un punto trascendental encontrado en esta población fue el conocimiento mecanizado y memorizado en cuanto al cepillado; se observó la influencia de los medios de comunicación y las campañas de promoción y prevención tradicionales. Los pobladores se encuentran muy enmarcados por el aspecto emocional, que puede verse reflejado en la presencia de un decaimiento general cuando se tiene sintomatología o patologías asociadas, refiriéndose a que estar triste es por la enfermedad. Conclusiones: Es relevante hacer énfasis en la importancia de una interacción directa con la comunidad, donde se pueda conocer a fondo todos sus aspectos sociales y además comprender las situación en la que se encuentran. De esta forma se podrían diseñar nuevas estrategias educativas por parte de los profesionales de la odontología, lo que podría contribuir a mejorar la calidad de vida de los pobladores. [González F, Carmona L, Agudelo Y, Campo P. Análisis social de las familias de] barrio Nelsón Mandela de Cartagena de Indias, en cuanto a sus conocimientos en salud y enfermedad general y oral. Ustasalud Odontologín 2006; 5: 115 - 122 ]
\end{abstract}

Palabras claves: Conocimientos en salud oral, Interacciones sociales, Higiene oral, Hermenéutica.

\section{SOCIAL ANALYSIS OF FAMILYS FROM NEISON MANDELA NEIBORHOOD AT CARTAGENA DE INDIAS AND THEIR KNOWLEDGE ABOUT ORAL HEALTH AND SICKNESS}

\begin{abstract}
objective: To analyze the knowledge referring to health and oral sickness that he population of Nelson Mandela of Cartageno de Indias has, having in mind the social situation which they belong to.

Materials and methods: Qualitative methodological focus, based on the hermeneutic method, applied on the population of Nelson Mandela. Ten families were selected belonging to social stratified level 1 and 2, corresponding to family nuclei with school children between ages 2 and 19 years. An individual survey was used to gather the information and a non participative observation on behalf of the people that were taking care of the children, selecting the most interesting interactions to fulfil the study objectives.

Results: A transcendental point found in this population was the memorized mechanical knowledge in reference to tooth brushing techniques, where it is noticeable the influence of communication means, the traditional health promotion and prevention campaigns, which do not really allow to know the procedures that they apply. It is also important to remark that these inhabitants are heavily marked by emotional aspects, that can be confirmed by a general sadness in their conduct when associated with a sickness or pathological symptoms; connecting sickness with sadness.

Conclusions: It is important to remark the importance of a direct interaction with the community where we can learn in depth all their social aspects and understand the situation in which they are. By this way, we can create new educational strategies on behalf of the future dentist, which could eventually lead to a diminishment of plaque indexes and a change of direction in their interest to maintain a good oral health. If they have the sufficient knowledge they can change their social level which can contribute to a better life quality.
\end{abstract}

Key Words: Knowledge in oral health, Social interactions, Oral hygiene, Hermeneutic. 


\section{INTRODUCCION}

En referencia a la salud y enfermedad oral, hay suficiente evidencia que propone a la prevención como la mejor forma de evitar problemas mayores relacionados con las enfermedades. Por lo tanto, en todos los contextos se ha reconocido la importancia de la promoción de la salud y la prevención de la enfermedad en cada una de las comunidades, es por esta razón que se busca que en cada población se diseñen opciones educativas innovadoras que modifiquen la forma convencional de realizar cada una de estas acciones, ${ }^{1,2}$ llegando más al fondo del problema y ante todo comprendiendo el por qué de estas situaciones.

En la ciudad de Cartagena se encuentran pobladores con circunstancias muy particulares que ameritan ser objeto de estudio; entre estos, se encuentra el barrio Nelson Mandela donde se presentan innumerables dificultades socio económicas, debido, posiblemente, a su condición de desplazados, que en muchos casos no tienen acceso a un trabajo y a una vivienda digna, evidenciándose precarias condiciones de salud general y oral. Desde esta perspectiva, al ser considerada la salud como un estado completo de bienestar físico, mental y social, y de acuerdo con el concepto de salud como decreto humano fundamental, se destacan determinados prerrequisitos para su consecución, entre los cuales se incluyen la paz, adecuados recursos económicos y alimenticios, vivienda, un ecosistema estable y un uso sostenible de los recursos. ${ }^{3}$

Bajo estas condiciones, en esta población se han realizado estudios epidemiológicos de caries dental en el 2004, encontrándose una prevalencia de $97.5 \%$ y unos factores de riesgo abordados desde el nivel de conocimiento que poseen estos habitantes sobre la salud y enfermedad oral. De esta forma, se intentó reconocer la influencia de las condiciones sociales desde el aspecto socioeconómico y educativo en la apropiación de las herramientas preventivas para erradicar la enfermedad. Sin embargo, con este estudio se observaron dificultades durante el análisis de los datos, por la imposibilidad de conocer el origen de la información e interpretar las conceptualizaciones que los habitantes manejan dentro de los factores de riesgo sociales que pueden estar asociados a la enfermedad. ${ }^{4}$

La identificación de este problema puede contribuir a reflejar las fallas en los programas de promoción de la salud y prevención de la enfermedad en la población, ya que durante décadas se ha venido enseñando salud oral a estas comunidades de alta vulnerabilidad, sin tener éxito al momento de lograr incidir en sus conocimientos. De esta forma, se han dejado de lado las necesidades de los pobladores, sin que el profesional de la salud se apropie del tema y se adentre en la comunidad para conocer de cerca sus necesidades y comprender el por qué los individuos no realizan las practicas orales recomendadas para evitar la enfermedad. ${ }^{5}$

Desde esta perspectiva, comprender lo humano es interpretar una acción percibida desde dentro, desde el punto de vista de la intención que la anima. Por lo tanto, es necesrio precisar que este modo de acercarse a los sujetos significa introducirse en el mundo de los sentidos expresado a través de sus acciones, precisamente porque la acción humana "al liberarse de su agente, adquiere una autonomía semejante a la autonomía semántica de un texto; deja un trazo, una marca; se inscribe en el curso de las cosas y se vuelve archivo y documento". ${ }^{6}$

Tomando como fundamento el constructivismo pedagógico donde el conocimiento y el aprendizaje, son el producto de una construcción mental, en la cual el fenómeno real se produce mediante la interacción sujeto cognoscente-objeto conocido, es inapropiado la separación entre investigador e investigado, ya que tanto los datos como los hechos científicos surgen de la interacción ininterrumpida del hombre frente a su entorno. ${ }^{7-10}$

Adicionalmente, el trabajo se enmarca desde la odontología que se ha realizado al interior de las comunidades, ya que se ha trabajado más en el tratamiento rehabilitador que en la promoción de la salud y la prevención de la enfermedad. De esta forma, se hace necesario que se implemente el uso de otros modelos pedagógicos, donde se intente conocer a la comunidad para que a partir de éstos se pueda brindar la oportunidad de modificar sus conocimientos y ponerlos en práctica desde sus condiciones económicas, sociales y emocionales.

Esta propuesta investigativa se relaciona con la posibilidad de conocer más a fondo a la población objeto de estudio y poder interpretar los comportamientos que se evidencien, para construir nuevas herramientas dentro de la prevención con métodos construidos a partir de la comunidad, con la comunidad y para la comunidad; siendo trascendente para la transformación que puede causar el interactuar ante factores institucionales, sociales y conceptuales. ${ }^{11}$

El objetivo de este estudio fue analizar los conocimientos en salud y enfermedad oral que tiene la población de Nelson Mandela en Cartagena de Indias, teniendo en cuenta la situación social en la que se encuentran, utilizando la hermenéutica como punto de partida para la interpretación del lenguaje social.

\section{MATERIALES Y MÈTODOS}

Este trabajo esta basado en un enfoque metodológico cualitativo, utilizando la hermenéutica, con base a descripciones de vivencias para profundizar en el problema de la representación del mundo. Se trabajó con base en un lenguaje descriptivo que tiene el propósito de hacer evidente la experiencia humana a través de la reflexión y así descubrir las formas genuinas y verdaderas de los propios pensamientos ${ }^{11}$ buscando conocer el pensamiento de los pobladores y de igual forma tratar de interpretar los fundamentos utilizados para la realización de algunas actividades sociales que promueven la salud y la poca apropiación de las acciones preventivas que se implementan a través del profesional de la odontología.

\section{6}


La metodología cualitativa da la posibilidad de observar y registrar los fenómenos y eventos sociales que intervienen en el proceso de apropiación del conocimiento que se instaura constantemente en los procesos educativos de los individuos. ${ }^{12-14}$ Se otorga una importancia a la subjetividad, integrándose el investigador a la cotidianidad, al lado del sujeto investigable, permitiendo que se exprese de sí mismo y encuentre en el diálogo el medio que permita tener una mayor aproximación al saber popular. ${ }^{11}$

En este sentido, se interactuó con la población de Nelson Mandela seleccionando a diez familias de estrato 1 y 2 correspondientes al núcleo familiar de niños escolarizados en edades de 2 y 19 años, por medio de la escuela Bherta Sunner, teniendo como visión las interacciones emanadas desde la escuela, el hogar y las actividades sociales por fuera de estos dos escenarios (diversión, deportes, juegos etc.).

Las categorías analizadas fueron: salud y enfermedad general, salud y enfermedad oral, prevención de la enfermedad oral, conocimiento sobre tratamientos orales, hábitos de salud oral, prácticas sociales.

La recolección de la información consistió, en primera instancia, en la observación del entorno en que se desenvuelve esta comunidad, describiendo las características que pueden ser desmotivantes para adquirir hábitos higiénicos orales, centrándose directamente en la observación de las vías de acceso, atención médica, servicios públicos, educación, recreación y centros culturales. Este aspecto permitió contar con un registro estructurado sobre ciertos elementos básicos para comprender la realidad humana objeto de análisis y focalizar la atención de la etapa de análisis en profundidad. Luego se realizó un mapeo al interior de los pobladores del sector, con el fin de seleccionar y hacer el encuadre de las diferentes interacciones que podrían aportar información útil para el estudio. Posteriormente, se realizaron entrevistas individuales estructuradas, a diez personas responsables de suministrar la educación en salud oral al interior del hogar, población seleccionada teniendo en cuenta que estuvieran dispuestas a participar, sin importar el nivel de jerarquía familiar y sobretodo que fueran mayores de edad. Por último, se realizaron dos entrevistas a profundidad para afianzar datos correspondientes a las categorías emergentes. Para efectos del análisis no bastó sólo con registrar las ideas sino que también se examinó el contexto en que esas ideas aparecen, identificándolo, principalmente, por una manera de hablar. ${ }^{11,15}$

La información fue analizada durante todo el proceso, desde que se inició la observación del entorno hasta la realización de las entrevistas. Para la observación del entorno se tuvieron en cuenta las condiciones de las calles o vías de acceso, los sistemas de drenaje de aguas residuales, la presencia de farmacias, tiendas que pudiesen suministrar, elementos de uso específicamente oral, como cepillos, crema dental, o medicamentos esenciales; la atención medica, servicios públicos, educación, recreación, centros culturales. Para la realización de las entrevistas se utilizaron las categorías planteadas, las emergentes y el posterior cruce de información recolectada, en los diferentes sujetos, realizándolo de forma manual e identificando las similitudes en sus respuestas y a su vez las discrepancias. Estas entrevistas fueron analizadas mediante la realización de comentarios y triangulaciones creados a partir del racionamiento de cada una de sus respuestas para, posteriormente, integrar los conceptos en cuanto a un "todo".

\section{RESULTADOS}

\section{CONCEPTOS SOBRE SALUD Y ENFERMEDAD GENERAI}

Durante este análisis se encontraron respuestas adquiridas de múltiples fuentes donde se pueden resaltar los factores biológi$\cos$, los cuales han hecho que se interprete la salud y la enfermedad de acuerdo con los signos y síntomas clínicos. También, se pudo percibir que hay una gran importancia por el factor emocional, ya que éstos asocian la enfermedad con estados de tristeza, cansancio y otros factores anímicos.

La salud y enfermedad general desde lo biológico: Con respecto a "la salud" se encuentra un conocimiento enmarcado en la ausencia o no de signos y síntomas clínicos basados en el bienestar fisico del organismo, encontrándose opiniones como "iAh! porque, ósea por mí mismo, por lo que yo me sienta, porque yo creo que si, si yo tuviera algo yo dijera que me siento mal de alguna cosa como no tengo nada" (P: 2, E: 5). Esto se puede presentar debido a que en estos individuos ocurre un efecto directo del problema desde sus síntomas. Además, fue común encontrar en algunas personas una relación de salud con no estar enfermo, condicionada a unas visitas permanentes al médico para la prevención de enfermedades

Salud enfermedad desde lo emocional: En esta población, la salud y la enfermedad se percibieron como realidades bajo dos perspectivas, es decir, en algunos instantes tiene importancia orgánica y en otros es comportamental; esto se traduce hacia una reducción de la actividad física, tratando de buscar alternativas terapéuticas que justifican la visita al médico. Este comportamiento social indica la incapacidad que la enfermedad produce para realizar cualquier actividad, influyendo en el estado de ánimo, ya que la persona entra en una especie de decaimiento, volviéndose improductivo, rodeado de dificultades económicas, "Tener salud, es no tener deudas, estar tranquilo, bien" ( $P: 2$, $E$ : 1) luego empieza la depresión y la soledad que en última instancia se percibe con un cambio de comportamiento. La significación más común que esta población tiene con los conceptos de salud desde lo emocional esta determinada por un bienestar fisico y económico, atribuyendo cualquier alteración emocional con la ausencia de estas.

\section{CONCEPTOS SOBRE SALUD ENFERMEDAD ORAL}

En esta categoría se encontró que la mayoría de los individuos se encaminaron hacia el concepto biológico, describiendo las 
enfermedades orales por los signos y síntomas que presentaron, obviando las características estéticas como signo de gran importancia para la salud oral.

La salud y enfermedad oral desde lo biológico: Al analizar este fenómeno, se encontró que en muy pocos casos afecta la calidad de vida de los habitantes, al no ser incapacitante como en el caso de la enfermedad general, ni tampoco se ve involucrada la posibilidad de perder la vida durante una manifestación oral patológica, lo cual conlleva a que no haya una asistencia oportuna porque se piensa que las enfermedades orales no ponen en riesgo la vida. Se encontró que el individuo solo percibe la presencia de la enfermedad cuando observa cambios en la estructura del diente sean de color o de forma, lo que lleva a determinar que las personas solo perciben la patología oral cuando esta en un estado avanzado. En cuanto a los conceptos sobre patologías orales especificas como la gingivitis y periodontitis, son muy escasos, la explicación del fenómeno no es muy clara y esta determinada a partir de una aproximación desde lo cotidiano.

\section{CONCEPTOS SOBRE PREVENCIÓN}

En este sentido se encontró que los elementos de higiene bucal de mayor reconocimiento entre los pobladores son el cepillo y la crema dental, pero a pesar de manejar este concepto, no es utilizado permanentemente en el núcleo familiar y existen diferentes elementos que dominan en preferencia al momento de adquirirse, representados por la influencia del factor económico.

Elementos de higiene bucal: Los elementos de mayor conocimiento, en esta población, son el cepillo dental, la crema dental, en algunos casos, el enjuague bucal y en muy pocas ocasiones la seda dental. Además, también se utiliza el bicarbonato como herramienta de higiene. Se apreció el uso de métodos empíricos porque se tiene la concepción de que éstos producen blanqueamiento y por su consistencia abrasiva puede producir mayor remoción de placa (Figura 1).

Actitudes preventivas: Un aspecto muy importante dentro de esta subcategoría, fue el conocimiento que tiene la comunidad acerca de aquellos alimentos que pueden producir un riesgo para la salud; se describieron cierta cantidad de alimentos perjudiciales para la salud general, no obstante, estos no son asociados directamente con los daños que puedan causar en la cavidad oral.

En cuanto a la asistencia al odontólogo, se manifestó una asociación directa con la presencia en el puesto de salud del barrio. Las visitas están relacionadas con la enfermedad de la boca 0 con la enfermedad general, siendo relevante la presencia de dolor, como síntoma determinante, dejando de lado que las enfermedades de cavidad oral también son prevenibles. De esta forma, se apreció que para los pobladores es muy normal la presencia de dolor y destrucción dental (Figura 2 y 3).

Prácticas de higiene oral: Con respecto a la manera cómo los adultos y los niños se cepillan, se encontró la gran influencia de los medios de comunicación y las charlas tradicionales que implementan los profesionales de la odontología sobre el uso de técnicas de cepillado dental, "Los dientes de arriba hacia abajo, los de abajo hacia arriba y las muelitas en forma circular" ( $P: 22$, $E$ : 1). De esta forma, la comunidad en general tiene un concepto establecido como lo que se debe hacer, pero se puede asumir que este concepto es algo mecanizado y que probablemente no se esta poniéndolo en practica, de acuerdo con los momentos de interacción que se vivieron dentro de la población lo que fue reiterado por los altos índices de placa bacteriana detectados en un estudio previo. ${ }^{4}$

\section{CONCEPTOS SOBRE TRATAMIENTO DE LAS ENFER- MEDADES ORALES}

En cuanto al tratamiento de lesiones cariosas o "de muelas picadas" se encontró que la mayoría de las personas asisten al odontólogo, probablemente, ante la presencia de sintomatología, siendo relevante que la ayuda profesional en estos casos, es importante. En otros casos, se encontró el uso de procedimientos empíricos de carácter natural, pudiéndose presentar afecciones más complejas al permitir todo un proceso evolutivo de la enfermedad, dado que solo buscan eliminar la sintomatología dolorosa. "¿Qué se hace? Bueno, a mi a veces me duele las muelas me duelen los dientes, aquí hay un palo que se llama sangregao a eso se le quita una cáscara y masco ese palo y eso no me duele mas oíste, y los niños no le han dolido, ellos están mudando ahora, a ellos no le duele el diente." (P: 13, E: 6)

En lo que se refiere al tratamiento de los dientes deciduos en cuanto a la movilidad fisiológica del recambio, estos utilizan una practica tradicional en donde para ellos no es de importancia la asistencia al odontólogo porque en su mayoría prefieren sacarlos con un "hilito" dado a que han observado a lo largo del tiempo y por sus experiencias que estos dientes no presentan raíces, este pensamiento se basa, probablemente, en la cantidad de hemorragia que se observa cuando son extraídos: "cuando están flojitos y hay que sacárselos se le sacan con un hilito, eso no tiene patas ni nada eso es enseguida" (P: 18, E: 6).

En caso de sangrado de las encías "gingivitis" el tratamiento más común son los enjuagues de agua tibia con sal. Sin embargo, a esta enfermedad no se le da mucha importancia debido a que no es incapacitante.

\section{CONCEPTOS SOBRE HABITOS DE HIGIENE ORAI.}

Según la literatura y las conductas regulares, en cuanto a la frecuencia del cepillado del individuo, se han creado patrones de seguimiento donde se indica que deben ser tres veces al día como mínimo para gozar de una buena salud oral, además del uso de la crema dental, la seda, y en algunos casos el enjuague bucal si esta a su alcance. En este aspecto, se encontró que la población tiene definido cuantas veces debe cepillarse, pero esta actividad, generalmente, se realiza una vez al día, sin tener en

118

Ustasalud Odontología 2006; 5:115 - 122 González F y col. 
cuenta que no se le esta dando la importancia que tiene después de cada comida, practicándose como una situación rutinaria a una hora específica.

En cuanto al uso de la crema dental, se observó que la mayoría de los pobladores le da la importancia que ésta tiene, sin embargo la disponibilidad de la crema en sus hogares esta limitada al factor económico, lo cual conlleva a que se vean en la necesidad de utilizar otros elementos proveedores de limpieza: "Bueno, ahora mismo nada más estoy utilizando es eso, bueno cuando no hay crema dental utilizo jabón o bicarbonato." (P: 25, E: 2)

En relación al uso la seda dental, se encontró que la mayoría de las personas conocen este elemento, pero su uso es bastante reducido ya que la población tiende a desconocer su objetivo y a esto se le suma el factor económico. Por eso, se utilizan otros elementos caseros que la reemplazan: "Si pero, uso hilo de coser porque no tengo pa' comprar seda dental " ( $P: 26$, E: 4). Adicionalmente, no hay accesibilidad de ésta a la población:" No, no la uso, porque no la considero importante en la limpieza, mejor es el Listerine" (P: 27, E: 1). Se podría sugerir que la seda dental no esta teniendo la debida importancia y que, en cambio, el enjuague dental sí, tal vez porque deja una sensación de limpieza y frescura que la seda no produce.

\section{CONOCIMIENTOS SOBRE PRÁCTICAS SOCIALES}

Se encontró que esta es una población con muchas dificultades económicas y en condiciones de salubridad deficientes, al igual que en el aspecto cultural. Todo esto reflejado en que esta población es categorizada como un estrato socio económico 1 y 2 , donde habitan personas que viven en condiciones de pobreza, siendo muchas las necesidades que presentan. En general, la descripción de esta pobreza se refleja en sus viviendas, ya que casi en su totalidad son construidas con tablas, techos de hojas de zinc, en espacios reducidos y, generalmente, su distribución esta dada por una o dos habitaciones, una sala, cocina y en muchos de los casos no están bien estructuradas, sino que se encuentra un lugar improvisado para tal fin; los pisos son de tierra y algunas de las familias presentan condiciones de hacinamiento. En cuanto a los servicios públicos, se encuentra el servicio de agua y luz, la cabeza de familia o jefes del hogar son, generalmente, personas que no terminaron su básica primaria y si lo hicieron no culminaron su secundaria o bachillerato, lo que conlleva a que el sustento económico se dé gracias al "rebusque" o al comercio informal que en muchas ocasiones no alcanza para solventar las necesidades materiales de su hogar. Además, al no existir un trabajo estable son beneficiarios del régimen subsidiado en algunos de los casos (Figura 4 y 5 ).

Esta observación permitió obtener un complemento de la información recolectada en las entrevistas, reflejando que, aunque la población frente a la participación en el proceso investigativo se mostró muy atenta, no se esta creando un ambiente en el que la comunidad solucione sus deficiencias, desde el punto de vista ambiental o del lugar que los rodea para que generen un cambio desde lo que esta a su alcance, ya que son pocos los pobladores que pertenecen a algún grupo comunitario que busque generar cambios, sus respuestas fueron "Si estoy en una campaña para hacer limpieza" (P: 28, E: 3).

En cuanto al aspecto deportivo y cultural, se encontró que aunque en la población no se observa un escenario apto para el esparcimiento de los habitantes de la zona, comentaron que pertenecen a grupos deportivos, culturales donde realizan actividades como la danza o las manualidades. Esto puede ser de gran importancia para la población joven ya que permite que tengan la mente más abierta ante los cambios y evita que encuentren entretenimiento en actividades inoficiosas que no generen cambios productivos a la sociedad (Figura 6).

Es importante mencionar que la población es conciente que hay muchas cosas por hacer para el mejoramiento de su entorno, ya que se encontraron respuestas como "Si señor por lo menos, ayudar a arreglar las calles, mantener las calles que no tengan tanto hueco, uno mismo es el que tiene que hacer eso porque el gobierno no esta haciendo eso" o "si, ósea la seguridad" siendo confirmado esto con la observación que confirmó que el ingreso a esta comunidad se da por una sola vía, la cual al igual que todas sus calles se encuentran sin pavimentar; el acceso sólo se da por una ruta de servicio urbano. Además de las deterioradas vías de acceso, se suman los desagües que se dan en las cunetas que bordean éstas, donde se aprecia un factor de riesgo ambiental para enfermedades infecciosas por el cúmulo de agua en descomposición, que entra en contacto con la población menor, sumándole a esto que en muchas zonas no se evidencia alumbrado público lo cual es un factor importante para el beneficio de la delincuencia organizada que aqueja permanentemente a toda la población (Figura 7).

\section{DISCUSIÓN}

Los pobladores del barrio de Nelson Mándela, son considerados una población pobre y vulnerable, con infinidades de necesidades económicas, de vivienda, vestido, cuidados en salud, de una salud física, mental, social, de una salud oral, estética y funcional.

Esta población tiene muchas concepciones divergentes en cuanto a la salud. Se observó que el factor emocional es relevante, en lo que tiene que ver con la presencia o no dela enfermedad, ya que se asocia a la enfermedad como causa de un decaimiento general, el cual produce tristeza y desanimo; estos elementos pueden estar condicionados por la situación social en la que se encuentran los pobladores, ya que siendo victimas del desplazamiento forzado, tienen un estilo de vida diferente, enmarcado por la perdida de una identidad cultural y social, lo que ocasiona diferentes formas de asumir los problemas representados por la falta de dinero y más que todo por el poco afecto que reciben del ciudadano en general, lo que lleva a que se les mire como responsables de su propia desgracia, al haber tomado parte en el conflicto interno del país, 


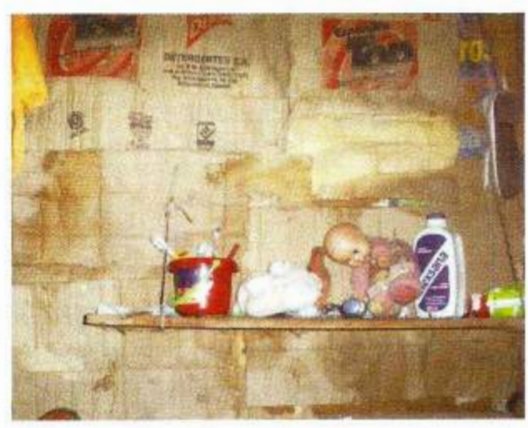

Figura 1. Almacenamiento de los cepillos dentales en el interior de la habitación.

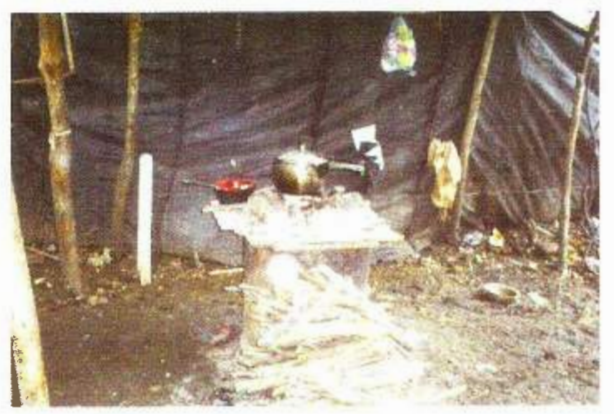

Figura 2. Medio utilizado por la mayoría de los pobladores para la preparación de los alimentos.

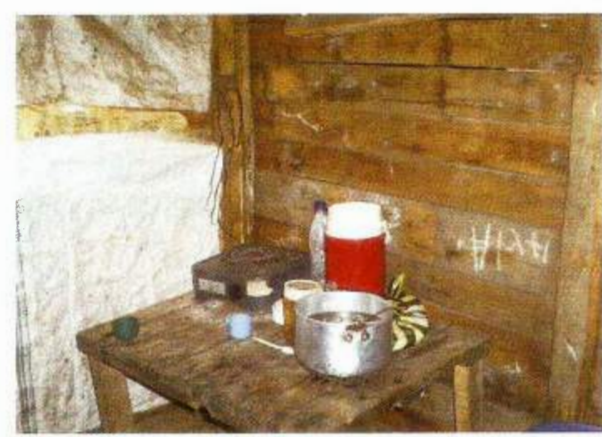

Figura 3. Uso de estufas eléctricas, para cocción de alimentos.

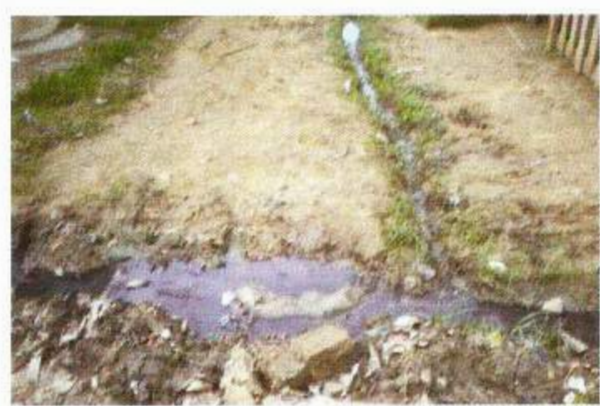

Figura 4. Concentración de aguas residuales alrededor de las vías de acceso de la población.

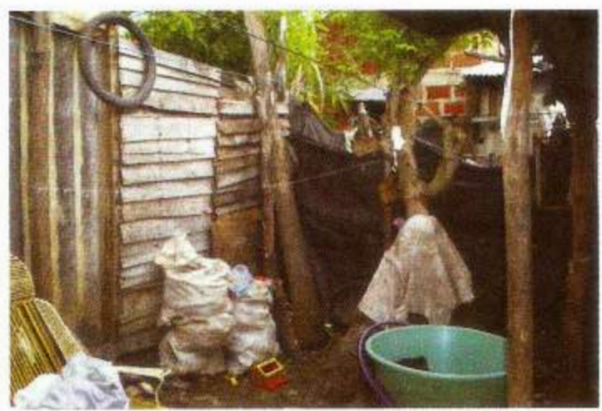

Figura 5. Almacenamiento de basuras al interior de la casas.

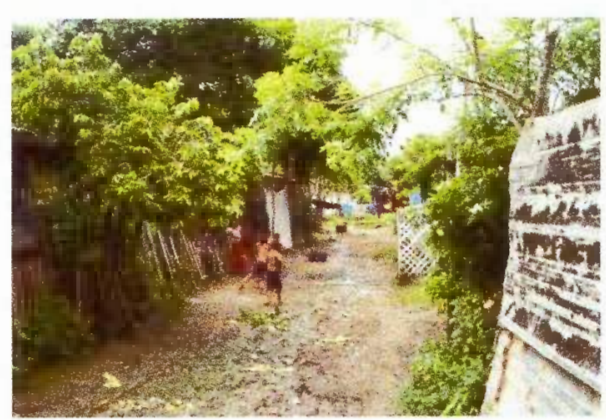

Figura 6. Niños en actividades lúdicas en contacto con factores de riesgo ambientales.

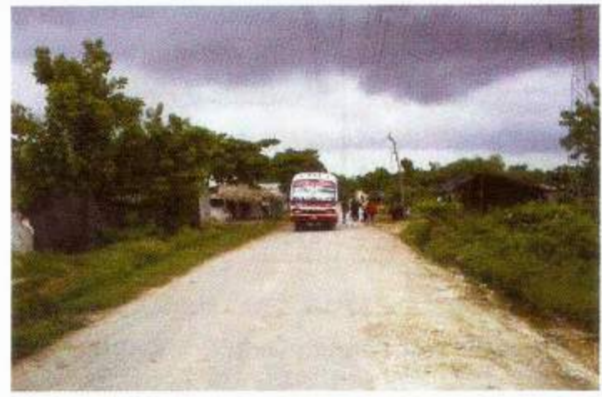

Figura 7. Medio de trasporte utilizado por la población para su desplazamiento.

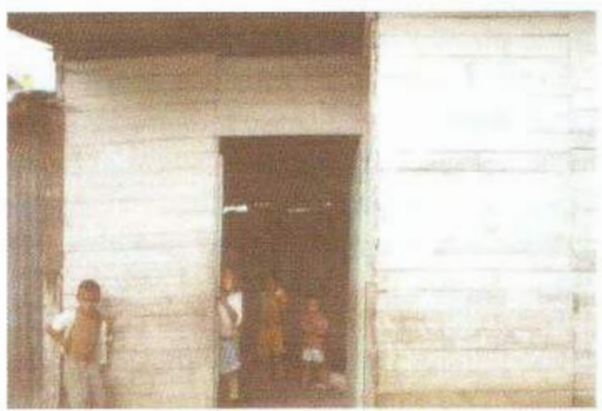

Figura 8. Condiciones de muchas viviendas de la población, estructuradas en madera. 
teniendo como limitaciones la capacidad para volver a levantarse desde el ámbito emotivo y lograr hacer nuevas amistades en un lugar digno para vivir. En este sentido, sienten que están alquilados en una tierra ajena y sin futuro, teniendo siempre la esperanza de volver a su lugar de origen, pero el temor hace que se acentúe el problema social en el que están inmersos..$^{16,17}$

En cuanto a lo económico, se encontraron limitaciones frente a la asistencia odontológica, para la adquisición de elementos de higiene oral, lo cual genera que esta población no utilice en su practica oral diaria la seda dental y mucho menos el enjuague bucal. A este respecto, se puede reiterar que esta población no se puede llegar con una practica odontológica tradicionalista, con actividades de promoción y prevención trasmisionistas, dedicadas a indicar lo que se debe usar para poder evitar las enfermedades orales, sin tener en cuenta si estos individuos están en la disposición económica y emotiva de adquirir estos elementos dentro de sus necesidades básicas. De esta forma, se hace fundamental empezar por ingresar como profesional dentro de su ambiente social y tratar de utilizar estrategias constructivistas innovadoras que permitan obtener información desde una perspectiva cualitativa para que la población supere las limitaciones cognitivas y emocionales que tiene, involucrando directamente al núcleo familiar desde todos los lideres comunitarios. De esta forma se podría llegar con una estrategia más exitosa como profesionales de la salud que somos, integrando los pilares fundamentales de una intervención comunitaria desde el mismo quehacer investigativo (Figura 8).

Con respecto a los problemas orales de la comunidad, se encontró que la presencia de signos y síntomas en cada uno de los pobladores en especial en lo relacionado con la cavidad oral, pueden ser determinantes de enfermedad, lo que conlleva a que dentro de la población no se perciban las actividades preventivas como mecanismo para evitar estos cambios desde lo biológico. De esta forma, se intenta interpretar el por qué son más útiles las actividades de recuperación de la salud y rehabilitación utilizadas en estados avanzados de la enfermedad, o en su defecto durante el uso de tratamientos empíricos. En este sentido, se puede reconocer que la población desconoce las condiciones óptimas de una buena salud oral y relacionan el bienestar con ausencia de las patologías que probablemente los han afectado, centrándose a nivel oral en la caries dental como la principal causa de perdida de dientes. Sin embargo, se aprecia que estos individuos no presentan un conocimiento muy claro sobre ésta enfermedad, siendo muchas veces asociada a otras patologías como la inflamación gingival.

\section{CONCLUSIONES}

Es importante, entonces, la interacción directa entre los profesionales de la salud y la comunidad, con el fin de conocer a fondo todos sus problemas, su origen y significados, tratando de comprender la situación social en la se encuentran, para poder trabajar desde una interacción social directamen- te relacionada con un lenguaje simbólico, a través de un intercambio de saberes. En este aspecto, es necesario que la Universidad de Cartagena, utilice estas herramientas desde lo institucional a nivel de todas las actividades de proyección social que están programadas, como una estrategia educativa para que los futuros profesionales vayan erradicando la práctica preventiva tradicionalista y observen al paciente desde una perspectiva integral, lo que conllevaría posiblemente a la disminución de las enfermedades orales y a un cambio comportamental desde el punto de vista social y humano del cual todos hacemos parte y tenemos el deber de contribuir a su mejoramiento continuo.

Desde esta perspectiva es importante que en Ias próximas investigaciones que se realicen en esta población se incluya la modalidad de investigación acción participativa, que lograría integrar este análisis a la práctica de la promoción de la salud y la prevención de la enfermedad con nuevas herramientas que podrán generar un cambio en la actitud de la población a partir de sus conocimientos y su disponibilidad socio económica, lo cual se vera reflejado en la disminución de las enfermedades orales.

Agradecimientos: A la comunidad del Barrio Nelson Mandela por su continuo y permanente apoyo para la consecución de los objetivos de este estudio. A la Facultad de Odontología de la Universidad de Cartagena, por su compromiso social al enfocar su trabajo comunitario hacia estas poblaciones vulnerables.

\section{BIBLIOGRAFIA}

1. República de Colombia. Ministerio de Salud. Estudio Nacional de Salud Bucal ENSAB III. Serie de documentos técnicos. 1998. p. 81.

2. Pareda C. Walter actis. c/luna, $11-1^{\circ}$ dcha. 28004 Madrid; 2003. P. $1-2$.

3. Yague M. Educación Sanitaria para la Promoción de la Salud. Organización Mundial de la Salud. p. 1.

4. Hoyos E, Martínez D, Quintana M, Padilla G, González, F, Carmona, L, Pérez, G. Prevalencia de caries "Ekstrand" y factores de riesgo en niños escolarizados entre 5 y 13 años del barrio Nelson Mandela Cartagena de Indias 2004. Memorias XV Encuentro Nacional de Investigación odontológica Universidad El Bosque Bogotá 2004.

5. Mercado-Martínez FJ. Investigación cualitativa en América Latina: Perspectivas críticas en salud. Internacional Journal of Qualitative Method 2002; 1: 1- 27. URL disponible en: http://www.ualberta.ca/ 〜iiqm/backissues/1_1Final/pdf/mercadospanish.pdf

6. Ríos T. La hermenéutica reflexiva en la investigación educacional. Revista Enfoques Educacionales 2005; 7: 51- 66. URL disponible en: http://csociales.uchile.cl/publicaciones/enfoques/09/ Rios_N7_2005.pdf

7. Anca MA. Fundamentos teóricos del constructivismo pedagógico. URL disponible en: http://www.monografias.com/trabajos27/ constructivismo-pedagogico/constructivismo-pedagogico.shtml

8. Glatthorn A. Constructivismo: Principios Básicos. Investigación y Práctica Educativa.1997. www.unam.mx/2001/1997/may97/42.gif. 
9. Logoeiro E. Métodos y Teorías Pedagógicas. URL disponible en: http://www.eduquenet.net/teoriasped.htm

10. Flórez O. Hacia una pedagogía del conocimiento. Editorial McGraw-Hill 1998; p. 159.

11. Sandoval C. Investigación cualitativa, módulos Icfes, Especialización métodos y técricas de investigación social, 2003.

12. Pita Fernández S, Pértigas Díaz S. Investigación cuantitativa y cualitativa. Cad Aten Primaria 2002; 9: 76 - 78. URL disponible en: http://www.fisterra.com/mbe/investiga/cuanti_cuali/ cuanti_cuali2.pdf

13. Lockett MO. La investigación cualitativa en Ciencias de la Salud. Una aproximación teórica. URL disponible en: http://odn.unne.edu.ar/ locketn/ma.doc

14. Caricote N. Como hacer investigación sin complicaciones. Acta Odontológica Venezolana 2004; 42: 3. URL disponible en: http:// ww w. actaodontologica.com/42_3_2004/ investigacion_sin_complicaciones.asp

15. Cabrero J, Martínez MR, Metodología de la Investigación. Técnicas de recogida de datos. URL disponible en: http://perso.wanadoo.es/ aniorte_nic/apunt_metod_investigac4_9.htm

16. Nieva B, Jácome $S$. Representaciones sociales del proceso saludenfermedad oral en poblaciones urbano marginales y su relación con los discursos y las prácticas institucionales. Primera parte. Revista de la Federación Odontológica Colombiana 1999; 57.

17. Nieva B, Jácome S. Representaciones sociales del proceso saludenfermedad oral en poblaciones urbano marginales y su relación con los discursos y las prácticas institucionales. Segunda parte. Revista de la Federación Odontológica Colombiana 1999; 58. CORREOS DE COLOMBIA

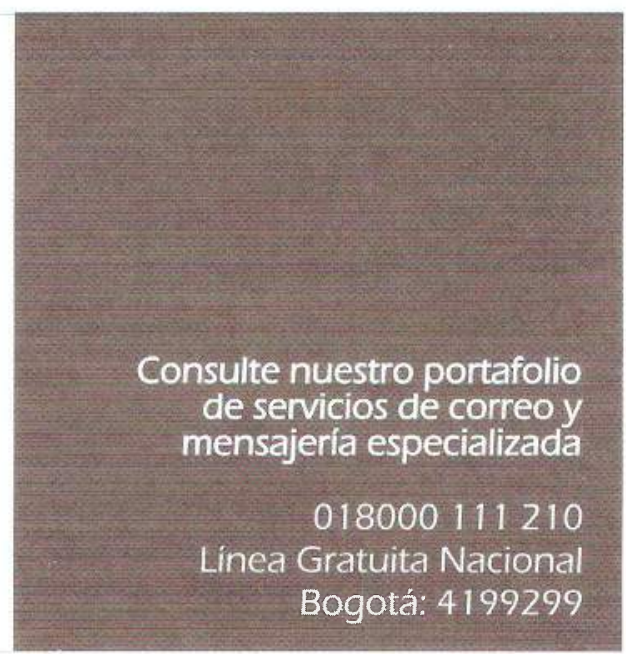

